\title{
Evolution of Automated Peritoneal Dialysis Machines
}

\author{
Anna Giuliani ${ }^{a}$ - Carlo Crepaldi ${ }^{a}$ - Sabrina Milan Manani ${ }^{a} \cdot$ \\ Sara Samonia ${ }^{\text {- Manuela Cannone }}$ - Massimo De Cal ${ }^{a, b}$ • \\ Claudio Ronco ${ }^{a-c}$
}

a Department of Nephrology, Dialysis and Transplantation, San Bortolo Hospital, Vicenza, Italy; ${ }^{\mathrm{b}}$ International Renal Research Institute of Vicenza (IRRIV), Vicenza, Italy; ' University of Padova, Padova, Italy

\begin{abstract}
Peritoneal dialysis (PD) has undergone several improvements over the years. Among the numerous advances, we may recall the improvement in the quality of fluids, safety of catheters and connections, knowledge of the peritoneal membrane in the process of mass transfer separation typical of PD. In parallel with these achievements, PD techniques have also displayed significant improvements mainly due to the evolution of machines and cyclers. Originally, bottles or containers were used to deliver and drain fluid to and from the peritoneal cavity by gravity using manual techniques. Subsequently, the development of semiautomatic or automatic machines have permitted to deliver an adequate treatment during night-time without the need of patient or care giver intervention. These advances solved the problem of treatment delivery, but other aspects including complications and adherence to prescription could only be managed using magnetic cards containing data from different treatments and brought by the patient at the following routinely planned hospital consultation. Today these limitations have been overcome by the new cycler "HOMECHOICE CLARIA" equipped with SHARESOURCE software featuring a bidirectional communication protocol that allows a full remote patient management (RPM). RPM has demonstrated significant advantages including higher technique survival, reduced rate of complications, and reduced costs in patients undergoing long-term PD.




\section{Introduction}

The utilization of automated peritoneal dialysis (APD) increased progressively from its original application, reaching 45\% of the cases in 2008 in developed countries [1]. In Italy in 2016, it has been reported in $50 \%$ of patients treated by $\mathrm{PD}$ [2]. This represents a consequence of continuous evolution of PD cyclers. Since the first description by Boen et al. [3, 4] in the early 1960s, PD cyclers have undergone significant improvements making APD a fast-growing modality for renal replacement therapy (Fig. 1). The success of APD derives from multiple factors. Automation has permitted to overcome some of the limitations of continuous ambulatory PD (CAPD), and has led to optimized management of ultrafiltration (UF) failure, achievement of adequate dialytic clearances in anuric patients, and avoidance of poor patient compliance. Moreover, APD is the preferred modality in young patients and in those with an active life, since it allows to be free from dialysis during the daytime. On top of these factors, technological improvement of cyclers was undoubtedly the most determinant element in the growth of APD. The ability of modern cyclers to deliver accurate and reliable treatments made APD a real alternative to CAPD. In the decade between 1970 and 1980, specific improvements were carried out in the hardware, moving from manual techniques to semi-automatic cyclers. Subsequently, automatic cyclers were designed for hospital use, capable of preparing the dialysis solution from tap water and liquid concentrates. More recently, in the 1990s, continuous progresses in the hardware component led to the availability of portable cyclers, suitable for home treatment. Further evolution regarded the user interface and software design. In particular, the use of memory magnetic cards made it possible to effectively control prescription, delivery and monitoring of different APD modalities. Furthermore, patient's card allowed to record treatments at home and review the adherence of patients when they were brought to the hospital for periodic consultation. Nowadays modern cyclers allow bidirectional communication between hospital and patient's home, through a cloud-based platform. This realtime monitoring represents an important component of telemedicine in the field of PD and allows for a true remote patient management (RPM) in out-of-hospital treated populations where medical surveillance remains essential.

\section{Origin of APD}

The first application of PD was in 1959 by Morton Maxwell. He set up a rudimental system to deliver fluids into abdomen, which can be considered the forerunner of the modern CAPD. The system consisted of two $1 \mathrm{~L}$ bottles, filled with 


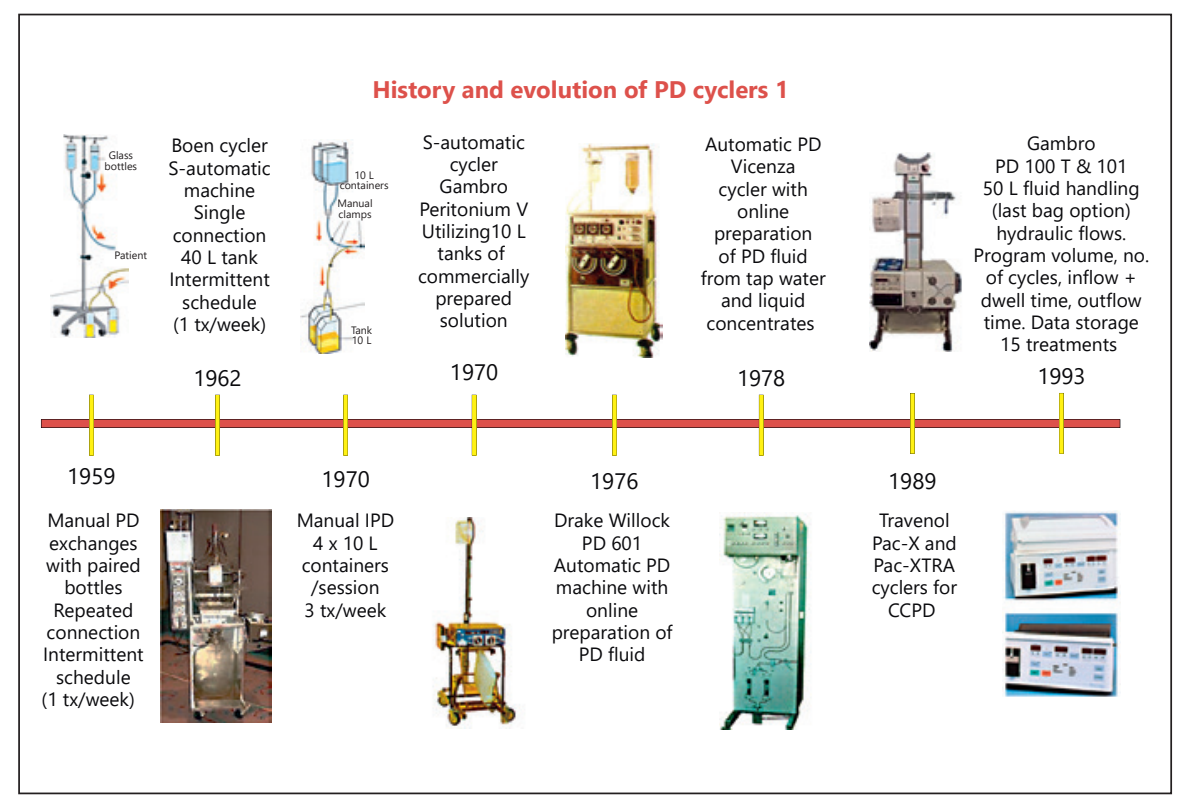

Fig. 1. Evolution of PD cyclers from origin to today. PD, peritoneal dialysis; $A P D$, automated PD; IPD, intermittent PD; CCPD, continuous cyclic PD.

warm dialysis solutions, connected through a Y tube, to the catheter. The bottles were hung above the bed, to allow the dialysis fluids to flow by gravity into the abdomen. After $1 \mathrm{~h}$ dwell time, the bottles were lowered into the floor and used for drainage. This procedure was repeated for $12-36 \mathrm{~h}$ and was performed manually. The maneuver of changing bottles requires multiple disconnections of the system, representing the major risk of touch contamination. To overcome this complication, containers of $10 \mathrm{~L}$ were subsequently used both for load and drain phases, significantly reducing the number of connections.

In 1962 in Seattle, Boen et al. [3, 4] conceived the first cycler able to deliver and drain fluids into and from the abdomen, semi-automatically. Dialysis fluids were prepared in the University pharmacy and stored in $40 \mathrm{~L}$ containers and periodically shipped to patient's home. The fluids were pumped into an elevated reservoir, filled up to $2 \mathrm{~L}$, and from there, moved to peritoneal cavity simply by gravity. Inflow, dwell, and outflow were regulated by a timer, which controlled clamps. Therapy was intermittent, performed once a week, over 20-22 h/session. The advantage of this semi-automatic technique, compared to the manual paired bottles, was the reduction of the number of connections and therefore the risk of touch contamination. Subsequently, smaller plastic containers (10 L) were connected in series for a closed-circuit PD. 
In 1966, Lasker designed the first "PD cycler." It featured 4 bottles of dialysis solution to obtain a reservoir of $8 \mathrm{~L}$, connected to pre-sterilized disposable tubings and bags. This concept of semi-automatic machine was then applied to many other cyclers with few variations. In principle, a pump was moving fluid to fill a $2 \mathrm{~L}$ bag suspended above the patient and then inflow and outflow into and from the abdomen was operated by gravity.

The next step in automatization was to design a machine able to produce sterile dialysate directly at patient's home, avoiding transport of large amount of fluids. At the end of 1960, Tenckhoff created a system able to purify cold tap water by reverse osmosis to be mixed with a liquid concentrate of glucose and electrolytes. The automatic cycler allowed the production of large amounts of safe and sterile dialysate, but it was bulky and costly. In 1970s, semi-automatic machines were mostly used in Europe, while automatic equipment was preferred in USA where $10 \mathrm{~L}$ tanks were not available.

Both semi-automatic and automatic machines began the era of APD and were mostly used in the hospital setting for intermittent PD, also defined nightly intermittent PD. With the advent of CAPD in the 1970s, the use of the machine was partially abandoned. CAPD was easier than APD and patients could manage treatment by themselves at home, without costly and cumbersome machinery. Moreover, CAPD was preferred because of its better results in terms of treatment adequacy [3,4]. This was the state of art until the 1980s when new generation cycler became available, spurring a renewed interest in APD.

\section{Evolution of Cyclers}

A renewed interest in APD occurred in the early 1980s when Diaz e Buzo described a new treatment schedule called continuous cyclic PD. The technique was performed through a simplified machine able to operate 3 exchanges during nighttime and one during daytime, with a schedule opposite to CAPD [5].

At the end of 1980s, cyclers underwent progressive improvement in terms of hardware components and layout, making APD safer, quieter, and less bulky. This is the case of Pac-X and its upgraded version Pac-XTRA produced by Baxter, PCS 2000 by Fresenius and PD 100 T from Gambro. This generation of cyclers utilized disposable materials and allowed individualization of dialysis by personalized prescription of intraperitoneal volume and dwell time. These machines allowed to perform different APD treatment schedules such as intermittent $\mathrm{PD}$, nightly intermittent $\mathrm{PD}$, continuous cyclic $\mathrm{PD}$, and even tidal volume prescription (TPD). 


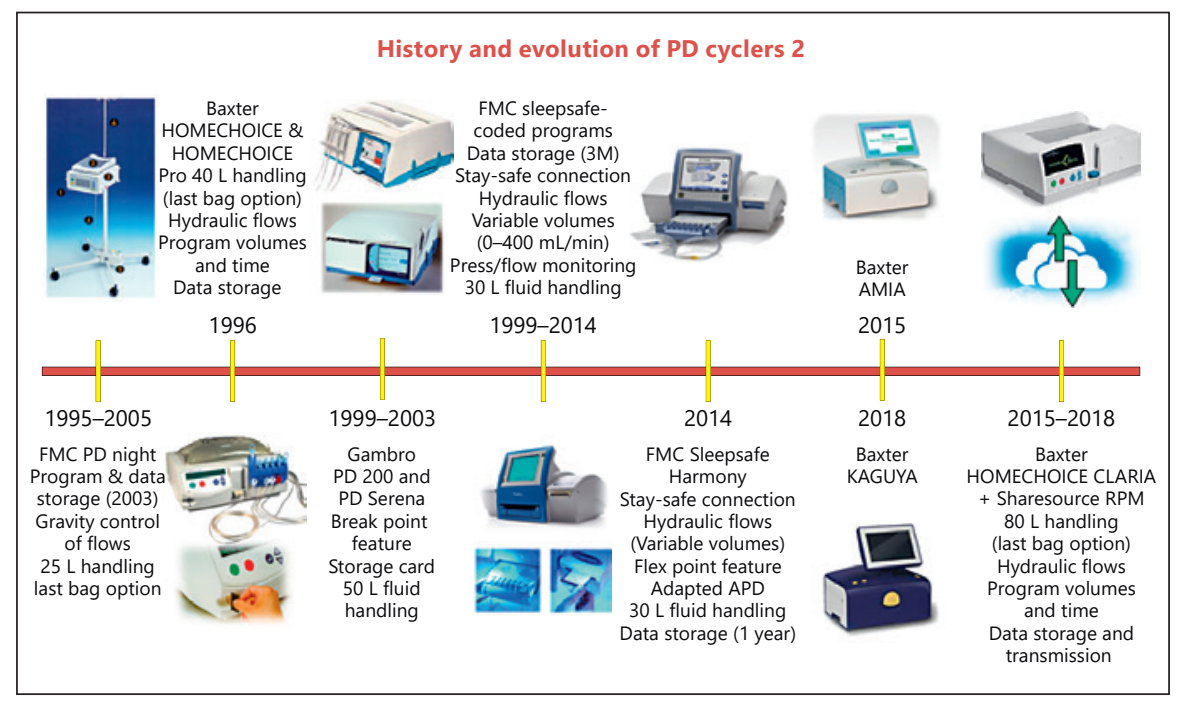

Fig. 2. Evolution of PD cyclers from origin to today. PD, peritoneal dialysis; $A P D$, automated PD.

A milestone occurred in 1994 when Baxter launched HOMECHOICE (Fig. 2). Thanks to improved hardware components, the new cycler was compact and portable, weighing only $12 \mathrm{~kg}$. Moreover, the device abandoned the gravity control of flows using volumetric pumps and allowing higher accuracy of dialytic exchanges. The following version, the HOMECHOICE Pro, added a $2 \mathrm{Mb}$ data card with 60 days of treatment storage capability. Memory cards revolutionized the way physicians and nurses manage therapy. Cards were permitted to program patient's prescription and to record treatment details. By inserting a card into the slot, prescription was uploaded by the machine and treatment memorized. For the first time, home therapy could be verified by analyzing possible problem experiences during treatment by patients. At the same time, patient's compliance and adherence to prescription could also be assessed. Data downloaded from the card during hospital visits were utilized to populate a data base containing important historical information on treatments and patients.

Other companies produced APD machine with similar features, such as the PD night equipment and its upgraded version, the Sleep Safe (FMC, Bad Homburg, Germany), the PD 100T, PD 101, PD 200 and Serena (Gambro, Lund, Sweden). The last version of all these machines presented similar features such as data recording (60-180 days of treatment), possibility to prescribe total dialysate volume, exchange fill volume, tidal percentage and time. Sleep Safe and Serena also allowed deciding the dwell time and number of cycles. Sleep Safe was equipped with a system for the automatic detection of the type of bag, avoiding possible mistakes in bag selection. It also allowed profiling glucose percentage at each cycle. All ma- 
chines were permitted to use solutions of other companies through adaptors. They were different in the way they moved and measured fluid volumes. Pneumatic and hydraulic pumps were used by HOMECHOICE Pro and Sleep Safe, respectively, while Serena incorporated a pressure chamber. Both HOMECHOICE Pro and Sleep Safe used a volumetric system to measure fluids, while Serena used a gravitybased system. In addition, Serena also allowed prescription in breakpoint modality, varying the tidal percentage depending on the drainage flow rate: when the drainage flow started to become too slow $(30-60 \mathrm{~mL} / \mathrm{min})$, a new fill cycle started, provided a pre-set cut off volume had been achieved. This modality optimized drainage and avoided spending too much time in the last part of the exchange to drain only $15-25 \%$ of the residual intraperitoneal volume.

All these cyclers were safe and accurate in delivering dialysis treatment. A built-in battery allowed to suspend treatment and memorize data in case of power breakdown. They were easy to use with a friendly user interface guiding the patient throughout the procedure, from therapy initiation to the final disconnection. With the evolution of information communication technology, cyclers were progressively equipped with specific software designed to individualize patient prescription based on personal data and treatment history. Last versions even included a section for simulation, based on mathematical models, of hypothetical adequacy targets obtained with different dialysis schedules in case of different peritoneal membrane characteristics $[6,7]$.

The most recent cyclers (HOMECHOICE CLARIA with the variants AMIA and KAGUYA, Baxter, Dirfield, IL, USA, and Sleep Safe Harmony, FMC, Bad Homburg, Germany) present significant innovation, mostly related to the software components (allowing new treatment schedules) and the bidirectional communication features (allowing true implementation of telemedicine and RPM).

Experimental studies were carried out to explore different areas of PD, including the possibility to perform continuous flow PD using a special double lumen catheter [8] and the potential development of wearable miniaturized cyclers for continuous wearable PD [9].

\section{HOMECHOICE CLARIA/SHARESOURCE Revolution}

A true revolution of PD occurred with the advent of real-time bidirectional communication between the cycler at patient's home and the hospital care team. This is today offered by the new HOMECHOICE CLARIA equipped with the cloudbased SHARESOURCE platform (Baxter, Dirfield, IL, USA). Physicians and nurses can verify and change the dialysis prescription by a simple login to the SHARESOURCE platform, while patients can stay out-of-hospital avoiding 
long and time-consuming travels to the PD center. There is no need to bring data cards for the periodic scheduled consultation since data are constantly supervised by the care team.

While patients can safely and comfortably continue their treatment at home, the hospital team supervises daily the performance of the cycler and the adherence to prescription, identifying any possible problem in real time. The possibility to see at-a-glance a series of parameters allows the care team to correct problems and verify the correspondence between prescribed and effectively delivered treatment doses. Information about treatment can also be analyzed and visualized as graphs and statistical trends for a period of interest. At the same time, change in prescription can be done remotely via computer and the patient only needs to accept and confirm the variation when he/she switches on the machine in his/her own home. The bidirectional communication helps to troubleshoot problems. Patients do not need to come to the hospital when a problem occurs with the machine, and the hospital team can rapidly correct and immediately verify the effect of a variation in prescription. All these aspects related to the HOMECHOICE CLARIA with Sharesource make a real revolution in PD allowing a true RPM [10-13].

RPM in PD is one important possibility in the area of telemedicine, improving the quality of the therapy and possibly expanding its application even in patients with lack of confidence or limited manual skills. RPM allows to reduce patient access to the center, affecting healthcare and personal cost. It may also reduce technique failure and patient drop out, increasing patient self-confidence with the home-based therapy. Finally interventions on dialysis schedules based on continuous treatment surveillance allow for a true personalization of therapy, in agreement with the most recent criteria of precision medicine $[12,13]$.

\section{Conclusion and Future Perspectives}

The remarkable expansion of automated PD has been mostly related to the technological improvement of modern cyclers that today are safe, compact, portable, and easy to use. With the parallel improvement of the dedicated software, the spectrum of PD techniques has been expanded leading to a personalized prescription case by case depending on the peritoneal membrane characteristics and results obtained from kinetic models and simulations. The combination of all these elements makes possible today to make a true RPM. Further advances and developments can be expected for the future. Hardware can be further miniaturized, possibly leading to wearable solutions. New biosensors will be developed to enable a fully integrated remote machine and patient monitoring pro- 
viding information on intraperitoneal pressure and operating a feedback on inflow and drainage kinetics. This will not only optimize exchange volumes and solute and water transport, but will also avoid short- and long-term complications. Moreover, biosensor could also be used to provide a turbidimetric measure of the effluent, avoiding unwanted delays in the diagnosis of peritonitis.

Software-guided prescription based on collected information will allow the cycler to optimize delivery of different PD modalities. Profiling the fill volume, glucose concentration, and dwell time will have a favorable effect on UF and solute transport in every single cycle [14].

Lastly, RPM could include more aspects of treatment, not just limited to the dialysis technique. Data on blood pressure and patient's weight will be recorded together with other information, possibly captured by imaging or video conferences [15]. All these efforts will be directed to make PD a true home-based therapy with minimum requirement of hospital visits or admission.

\section{References}

1 Jain AK, Blake P, Cordy P, Garg AX: Global trends in rates of peritoneal dialysis. J Am Soc Nephrol 2012;23:533-544.

2 Marinangeli G, Cabiddu G, Neri L, Viglino G, Corciulo R, Rocca AR, Laudon A, Finato V: [Peritoneal Dialysis in Italy: the fourth GSDP-SIN census 2012]. G Ital Nefrol 2017;34:110-137.

3 Boen ST, Mulinari AS, Dillard DH, Scribner BH: Periodic peritoneal dialysis in the management of chronic uremia. Trans Am Soc Artif Intern Organs 1962;8:256-265.

4 Boen ST, Mion CM, Curtis FK, Shilipetar G: Periodic peritoneal dialysis using the repeated puncture technique and an automatic cycling machine. Trans Am Soc Artif Intern Organs 1964; 10:409-414.

5 Diaz-Buxo JA: The place for automated peritoneal dialysis. Adv Perit Dial 1992;8:98-101.

6 Dell'Aquila R, Rodighiero MP, Spano E, Di Loreto P, Kohn CO, Cruz D, Polanco N, Kuang D, Corradi $\mathrm{V}$, De Cal M, Ronco C: Advances in the technology of automated, tidal, and continuous flow peritoneal dialysis. Perit Dial Int 2007;27(suppl 2):S130-S137.

\section{Claudio Ronco}

Department of Nephrology, Dialysis and Transplantation San Bortolo Hospital, Viale Rodolfi 37

IT-36100 Vicenza (Italy)

E-Mail cronco@goldnet.it
7 Corciulo R, Russo R: [Cyclers for APD: current potential and possible developments]. G Ital Nefrol 2009;26:355-368.

8 Ronco C, Amerling R: Continuous flow peritoneal dialysis: current state-of-the-art and obstacles to further development. Contrib Nephrol 2006;150: 310-320.

9 Ronco C, Fecondini L: The vicenza wearable artificial kidney for peritoneal dialysis (ViWAK PD). Blood Purif 2007;25:383-388.

10 Rosner MH, Ronco C: Remote monitoring for continuous peritoneal dialysis. Contrib Nephrol 2012;178:68-73.

11 Rosner MH, Ronco C: Remote monitoring for the wearable artificial kidney. Contrib Nephrol 2011; 171:243-247.

12 Milan Manani S, Crepaldi C, Giuliani A, Virzi GM, Garzotto F, Riello C, de Cal M, Rosner MH, Ronco C: Remote monitoring of automated peritoneal dialysis improves personalization of dialytic prescription and patient's independence. Blood Purif 2018;46:111-117.

13 Milan Manani S, et al: Longitudinal experience with remote monitoring for automated peritoneal dialysis patients. Nephron 2019;1-9.

14 Fischbach M, Zaloszyc A, Schaefer B, Schmitt C: Adapted automated peritoneal dialysis. Adv Perit Dial 2014;30:94-97.

15 Nayak KS, Ronco C, Karopadi AN, Rosner MH: Telemedicine and remote monitoring: supporting the patient on peritoneal dialysis. Perit Dial Int 2016;36:362-366. 